\title{
Nonclassical Properties of Para-Bose Superposition States
}

\author{
Y.-H. YU AND D.-M. WANG \\ Department of Applied Physics, Zhejiang University of Technology \\ Hangzhou 310014, P.R. China
}

(Received April 8, 2008)

\begin{abstract}
We investigate the nonclassical properties of para-Bose superposition states which involve the vacuum and one- or two-photon state of paraboson. It is found that these para-Bose superposition states exhibit stronger squeezing and antibunching. The amplitude-squared squeezing for these states is also examined.
\end{abstract}

PACS numbers: 42.50.Dv, 03.65.Fd

\section{Introduction}

It is well known that the nonclassical states of the light field play an important role in quantum optics. In the last decade, the nonclassical states which arise as the coherent superposition of two quantum states has attracted much attention [1-3]. In Ref. [1] Wódkiewicz et al. introduce a new kind of superposition states which exhibit squeezing fluctuations and open new possibilities of generating squeezed states.

In recent years, simulated by recent experimental observation of fractional quantum Hall effect $[4,5]$ in two-dimensional electron gas, much attention has been focused on various nontraditional statistics: fractional statistics [6-8] and parastatistics [9-11]. Parastatistics was first introduced by Green [9] as a generalization of the Bose and Fermi statistics. This generalization introduced trilinear relations in place of the bilinear relations that describe the Bose and Fermi systems. Recently, Greenberg and Mishra [11] have shown that the parastatistics can be quantized by using path integrals.

In this letter, we present two kinds of generalized para-Bose superposition states and investigate its nonclassical properties. It is shown that these para-Bose superposition states exhibit stronger squeezing and antibunching. The amplitude-squared squeezing for these states is also examined.

\section{Theory}

We first briefly outline the para-Bose oscillator [12]. The basic commutation relation is

$$
[\hat{a}, \hat{H}]=\hat{a},
$$

where

$$
\hat{H}=\frac{1}{2}\left(\hat{a}^{\dagger} \hat{a}+\hat{a} \hat{a}^{\dagger}\right) .
$$

The para-Bose number operator is defined as

$$
\hat{N}=\hat{H}-h_{0}=\frac{1}{2}\left(\hat{a}^{\dagger} \hat{a}+\hat{a} \hat{a}^{\dagger}\right)-h_{0},
$$

which leads to

$$
[\hat{N}, \hat{a}]=-\hat{a}, \quad\left[\hat{N}, \hat{a}^{\dagger}\right]=\hat{a}^{\dagger} .
$$

It is noted that $h_{0}$ is the lowest eigenvalue of $\hat{H}$ and $\hat{a}$ and $\hat{a}^{\dagger}$ are para-Bose annihilation and creation operators, respectively. For the special case $h_{0}=\frac{1}{2}$, the para-Bose oscillator reduces to the ordinary harmonic oscillator.

The para-Bose number states are the eigenstates of $\hat{N}$ and satisfy the following relations:

$$
\begin{aligned}
& \hat{N}|n\rangle_{h_{0}}=n|n\rangle_{h_{0}}, \\
& \hat{a}|2 n\rangle_{h_{0}}=\sqrt{2 n}|2 n-1\rangle_{h_{0}}, \\
& \hat{a}|2 n+1\rangle_{h_{0}}=\sqrt{2\left(n+h_{0}\right)}|2 n\rangle_{h_{0}}, \\
& \hat{a}^{\dagger}|2 n\rangle_{h_{0}}=\sqrt{2\left(n+h_{0}\right)}|2 n+1\rangle_{h_{0}}, \\
& \hat{a}^{\dagger}|2 n+1\rangle_{h_{0}}=\sqrt{2(n+1)}|2 n+2\rangle_{h_{0}} .
\end{aligned}
$$

We then consider the superposition state of vacuum and one-photon state of paraboson

$$
\left|\Psi_{1}\right\rangle=\alpha|0\rangle_{h_{0}}+\beta|1\rangle_{h_{0}}
$$

where $\alpha=|\alpha| \mathrm{e}^{\mathrm{i} \theta}$ and $\beta=|\beta| \mathrm{e}^{\mathrm{i}(\theta+\Delta \varphi)}$ are the probability amplitudes for zero- and one-photon excitation of paraboson, and $|\alpha|^{2}+|\beta|^{2}=1$. Now we study the squeezing properties of the para-Bose superposition state $\left|\Psi_{1}\right\rangle$. As usual, a pair of quadrature operators $\hat{X}_{1}$ and $\hat{X}_{2}$ in terms of the para-Bose creation operator $\hat{a}^{\dagger}$ and annihilation operator $\hat{a}$ can be defined as:

$$
\hat{X}_{1}=\frac{1}{2}\left(\hat{a}+\hat{a}^{\dagger}\right), \quad \hat{X}_{2}=\frac{1}{2 \mathrm{i}}\left(\hat{a}-\hat{a}^{\dagger}\right) .
$$

The variances $\left\langle\left(\Delta \hat{X}_{i}\right)^{2}\right\rangle \equiv\left\langle\hat{X}_{i}^{2}\right\rangle-\left(\left\langle\hat{X}_{i}\right\rangle\right)^{2}, i=1,2$ :

$$
\left\langle\left(\Delta \hat{X}_{1}\right)^{2}\right\rangle\left\langle\left(\Delta \hat{X}_{2}\right)^{2}\right\rangle \geq \frac{1}{4}\left|\left\langle\left[\hat{X}_{1}, \hat{X}_{2}\right]\right\rangle\right|^{2} .
$$

A state of the field is said to be squeezed when one of the quadrature components $\hat{X}_{1}$ and $\hat{X}_{2}$ satisfies the con- 
dition $\left\langle\left(\Delta \hat{X}_{i}\right)^{2}\right\rangle<\frac{1}{2}\left|\left\langle\left[\hat{X}_{1}, \hat{X}_{2}\right]\right\rangle\right|$. The squeezing can be measured by the squeezing parameters $Q_{i}, i=1,2$, defined by:

$$
Q_{i}=\frac{\left|\left\langle\left[\hat{X}_{1}, \hat{X}_{2}\right]\right\rangle\right|-2\left\langle\left(\Delta \hat{X}_{i}\right)^{2}\right\rangle}{\left|\left\langle\left[\hat{X}_{1}, \hat{X}_{2}\right]\right\rangle\right|},
$$

where $0<Q_{i} \leq 1$ for squeezing. For the operator $\hat{X}_{1}$ we find that the variance in the state is

$$
\begin{aligned}
& \left\langle\hat{X}_{1}\right\rangle=\sqrt{2 h_{0}}|\beta|\left(1-|\beta|^{2}\right)^{\frac{1}{2}} \cos \Delta \varphi, \\
& \left\langle\hat{X}_{1}^{2}\right\rangle=\frac{1}{2}\left(h_{0}+|\beta|^{2}\right), \\
& \left\langle\left(\Delta \hat{X}_{1}\right)^{2}\right\rangle=\frac{1}{2}\left(h_{0}+|\beta|^{2}\right)-2 h_{0}|\beta|^{2} \\
& \quad \times\left(1-|\beta|^{2}\right) \cos ^{2} \Delta \varphi .
\end{aligned}
$$

It then follows that:

$$
Q_{1}^{(1)}=\frac{4 h_{0}|\beta|^{2}\left(1-|\beta|^{2}\right) \cos ^{2} \Delta \varphi-2 h_{0}|\beta|^{2}}{h_{0}+\left(1-2 h_{0}\right)|\beta|^{2}} .
$$

Here $\Delta \varphi$ is the relative phase between $\alpha$ and $\beta$. In order to obtain stronger squeezing, we can take $\cos ^{2} \Delta \varphi=1$ in Eq. (11). Then Eq. (11) becomes

$$
Q_{1}^{(1)}=\frac{2 h_{0}|\beta|^{2}-4 h_{0}|\beta|^{4}}{h_{0}+\left(1-2 h_{0}\right)|\beta|^{2}} .
$$

It is not difficult to find the maximum squeezing $Q_{1 \max }^{(1)}=$ $\frac{2 h_{0}}{\left(1+\sqrt{2 h_{0}}\right)^{2}}$ when $|\beta|^{2}=\frac{\sqrt{h_{0}}}{\sqrt{2}+2 \sqrt{h_{0}}}$. For the special case $h_{0}=\frac{1}{2}$, the above results are in agreement with those obtained in Ref. [3].

\section{Results and discussion}

In Fig. 1 we plot $Q_{1}^{(1)}$ as a function of $|\beta|$ and $h_{0}$ for $\cos ^{2} \Delta \varphi=1$. From Fig. 1 we see that the squeezing in para-Bose superposition state $\left|\Psi_{1}\right\rangle$ increases with the parameter $h_{0}$. The numerical result for three different values of $h_{0}: h_{0}=0.2,0.5$ and 0.8 are shown in Fig. 2 for the squeezing parameter $Q_{1}^{(1)}$. It is quite clear from these figures that the squeezing in $\left|\Psi_{1}\right\rangle$ firstly increases with the parameter $|\beta|$, then decreases with $|\beta|$ after achieving a maximal value for a fixed $h_{0}$. We can observe that the field quadrature $\hat{X}_{1}$ exhibits a stronger squeezing over a wide range of parameters.

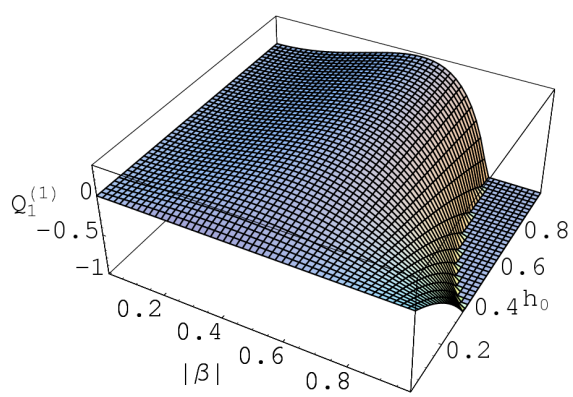

Fig. 1. The squeezing parameter $Q_{1}^{(1)}$ as a function of $|\beta|$ and $h_{0}$ for $\cos ^{2} \Delta \varphi=1$

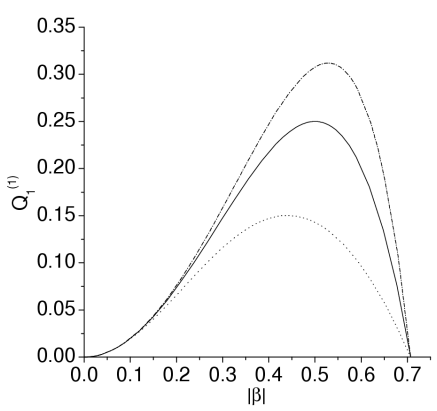

Fig. 2. The squeezing parameter $Q_{1}^{(1)}$ as a function of $|\beta|$ for three different values of $h_{0}=0.2$ (dotted line), 0.5 (solid line) and 0.8 (dashed-dotted line) with $\cos ^{2} \Delta \varphi=1$.

Next, we study two-photon para-Bose superposition state

$$
\left|\Psi_{2}\right\rangle=\alpha|0\rangle_{h_{0}}+\beta|2\rangle_{h_{0}} .
$$

By the same procedure used above, the squeezing parameter $Q_{1}^{(2)}$ can also be derived as follows:

$$
Q_{1}^{(2)}=-\frac{2}{h_{0}}\left[|\beta|^{2}+\sqrt{h_{0}}|\beta|\left(1-|\beta|^{2}\right)^{\frac{1}{2}} \cos \Delta \varphi\right] .
$$

In order to obtain strong squeezing, we can take $\cos \Delta \varphi=-1$ and then Eq. (14) reduces to

$$
Q_{1}^{(2)}=\frac{2}{h_{0}}\left[\sqrt{h_{0}}|\beta|\left(1-|\beta|^{2}\right)^{\frac{1}{2}}-|\beta|^{2}\right] .
$$

When $h_{0}=\frac{1}{2}$, Eq. (15) reduces to the result of Ref. [1]. It can be shown that the maximum squeezing is $Q_{1 \max }^{(2)}=\frac{1}{h_{0}}\left(\sqrt{1+h_{0}}-1\right)$ when $|\beta|^{2}=\frac{1}{2}\left(1-\frac{1}{\sqrt{1+h_{0}}}\right)$.

We plot the squeezing parameter $Q_{1}^{(2)}$ as a function of $|\beta|$ and $h_{0}$ in Fig. 3. It is shown in Fig. 4 that the squeezing parameter $Q_{1}^{(2)}$ as a function of $|\beta|$ for three different values of $h_{0}: h_{0}=0.2,0.5$ and 0.8. From these figures, we can see clearly that the squeezing in para-Bose superposition state $\left|\Psi_{2}\right\rangle$ also increases with the parameter $|\beta|$, then decreases after achieving a maximum value. However, the squeezing in para-Bose superposition state $\left|\Psi_{2}\right\rangle$ decreases with the parameter $h_{0}$ that is different from the situation in $\left|\Psi_{1}\right\rangle$.

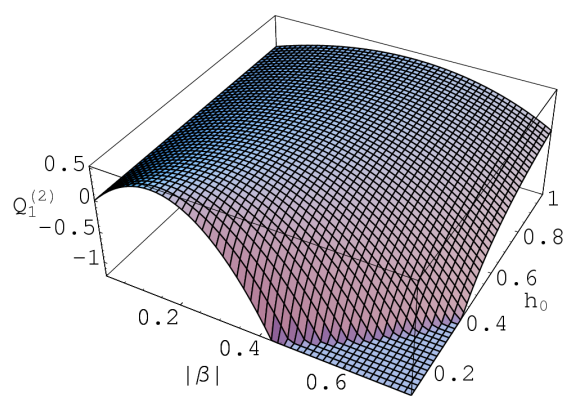

Fig. 3. The squeezing parameter $Q_{1}^{(2)}$ as a function of $|\beta|$ and $h_{0}$ for $\cos \Delta \varphi=-1$. 


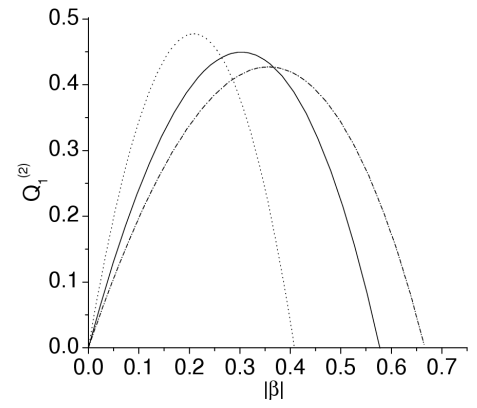

Fig. 4. The squeezing parameter $Q_{1}^{(2)}$ as a function of $|\beta|$ for three different values of $h_{0}=0.2$ (dotted line), 0.5 (solid line) and 0.8 (dashed-dotted line) with $\cos \Delta \varphi=-1$.

We then proceed to study the amplitude-squared squeezing for the vacuum and the one- or two-photon para-Bose superposition state. Hillery [12] introduced the concept of the amplitude-squared squeezing of the light field and noted that this kind of squeezing is also a nonclassical effect. The amplitude-squared squeezing is defined in terms of the following two operators:

$$
\hat{Y}_{1}=\frac{1}{2}\left(\hat{a}^{2}+\hat{a}^{\dagger 2}\right), \quad \hat{Y}_{2}=\frac{1}{2 \mathrm{i}}\left(\hat{a}^{2}-\hat{a}^{\dagger 2}\right) .
$$

It is easy to prove that $\hat{Y}_{1}$ and $\hat{Y}_{2}$ satisfy the commutation relation

$$
\left[\hat{Y}_{1}, \hat{Y}_{2}\right]=\frac{\mathrm{i}}{2}\left[\hat{a}^{2}, \hat{a}^{\dagger 2}\right],
$$

and the uncertainty relation

$$
\left\langle\left(\Delta \hat{Y}_{1}\right)^{2}\right\rangle\left\langle\left(\Delta \hat{Y}_{2}\right)^{2}\right\rangle \geq \frac{1}{16}\left|\left\langle\left[\hat{a}^{2}, \hat{a}^{\dagger 2}\right]\right\rangle\right|^{2} .
$$

Amplitude-squared squeezing is said to exist if

$$
\left\langle\left(\Delta \hat{Y}_{1}\right)^{2}\right\rangle<\frac{1}{4}\left|\left\langle\left[\hat{a}^{2}, \hat{a}^{\dagger 2}\right]\right\rangle\right|
$$

and can be measured by the parameters $Q_{i \mathrm{a}}(i=1,2)$,

$$
Q_{i \mathrm{a}}=\frac{\left|\left\langle\left[\hat{Y}_{1}, \hat{Y}_{2}\right]\right\rangle\right|-2\left\langle\left(\Delta \hat{Y}_{i}\right)^{2}\right\rangle}{\left|\left\langle\left[\hat{Y}_{1}, \hat{Y}_{2}\right]\right\rangle\right|},
$$

where $0<Q_{i \mathrm{a}} \leq 1$ for amplitude-squared squeezing.

It is easy to show that there does not exist the amplitude-squared squeezing for the vacuum and one-photon para-Bose superposition state. However, for the vacuum and two-photon para-Bose superposition state, we can find the variance of the operator $\hat{Y}_{1}$ as follows:

$$
\begin{aligned}
& \left\langle\left(\Delta \hat{Y}_{1}\right)^{2}\right\rangle=h_{0}+2\left(1+h_{0}\right)|\beta|^{2}-4 h_{0}|\beta|^{2}\left(1-|\beta|^{2}\right) \\
& \quad \times \cos ^{2} \Delta \varphi
\end{aligned}
$$

It then follows that:

$$
Q_{1 \mathrm{a}}^{(2)}=\frac{4 h_{0}|\beta|^{2}\left(1-|\beta|^{2}\right) \cos ^{2} \Delta \varphi-2 h_{0}|\beta|^{2}}{h_{0}+2|\beta|^{2}} .
$$

If we take $\cos ^{2} \Delta \varphi=1$, Eq. (22) reduces to

$$
Q_{1 \mathrm{a}}^{(2)}=\frac{2 h_{0}|\beta|^{2}-4 h_{0}|\beta|^{4}}{h_{0}+2|\beta|^{2}} .
$$

It is not difficult to find that the maximum amplitude-squared squeezing for the para-Bose superposition state
$\left|\Psi_{2}\right\rangle, \quad Q_{\text {1amax }}^{(2)}=\left(\sqrt{h_{0}^{2}+h_{0}}-h_{0}\right)^{2}$ when $|\beta|^{2}=$ $\frac{1}{2}\left(\sqrt{h_{0}^{2}+h_{0}}-h_{0}\right)$.

In Fig. 5 we show the parameter $Q_{1 \mathrm{a}}^{(2)}$ as a function of $|\beta|$ and $h_{0}$. The numerical result for three different values of $h_{0}: h_{0}=0.2,0.5$ and 0.8 is also displayed in Fig. 6 for the parameter $Q_{1 \mathrm{a}}^{(2)}$. It is noted that the amplitude-squeezing in $\left|\Psi_{2}\right\rangle$ is similar as the general squeezing in $\left|\Psi_{1}\right\rangle$, but it is smaller than the general squeezing in $\left|\Psi_{1}\right\rangle$.

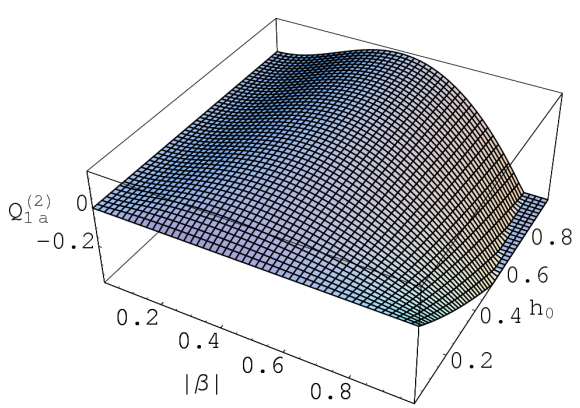

Fig. 5. The squeezing parameter $Q_{1 \mathrm{a}}^{(2)}$ as a function of $|\beta|$ and $h_{0}$ for $\cos ^{2} \Delta \varphi=1$.

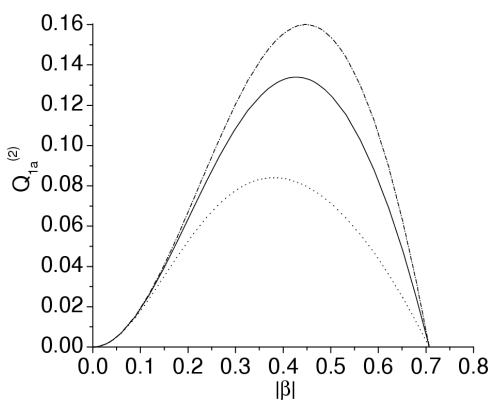

Fig. 6. The squeezing parameter $Q_{1 \mathrm{a}}^{(2)}$ as a function of $|\beta|$ for three different values of $h_{0}=0.2$ (dotted line), 0.5 (solid line) and 0.8 (dashed-dotted line) with $\cos ^{2} \Delta \varphi=1$.

Finally, we discuss the antibunching effect for the para-Bose superposition states $\left|\Psi_{1}\right\rangle$ and $\left|\Psi_{2}\right\rangle$. It is easy to prove that the para-Bose superposition state $\left|\Psi_{1}\right\rangle$ has zero degree of second-order coherence

$$
g^{2}(0) \equiv \frac{\left\langle\hat{a}^{\dagger} \hat{a}^{\dagger} \hat{a} \hat{a}\right\rangle}{\left\langle\hat{a}^{\dagger} \hat{a}\right\rangle^{2}}=0 .
$$

Therefore, we conclude that the state exhibits the antibunching effect for all values of $\beta$.

For the para-Bose superposition state $\left|\Psi_{2}\right\rangle$ has a nonzero degree of second-order coherence

$$
g^{2}(0)=\frac{h_{0}}{|\beta|^{2}} .
$$

The state $\left|\Psi_{2}\right\rangle$ exhibits the antibunching effect for $|\beta|^{2}>$ $h_{0}$, in dependence of the relative phase $\Delta \varphi$.

We plot the degree of second-order coherence $g^{2}(0)$ as a function of $|\beta|$ and $h_{0}$ in Fig. 7 , and as a function of 
$|\beta|$ for three different values of $h_{0}: h_{0}=0.3,0.5$ and 0.7 in Fig. 8. From these figures, we observe that the antibunching effect for the para-Bose superposition state $\left|\Psi_{2}\right\rangle$ increases with the $|\beta|$ and decreases with the $h_{0}$.

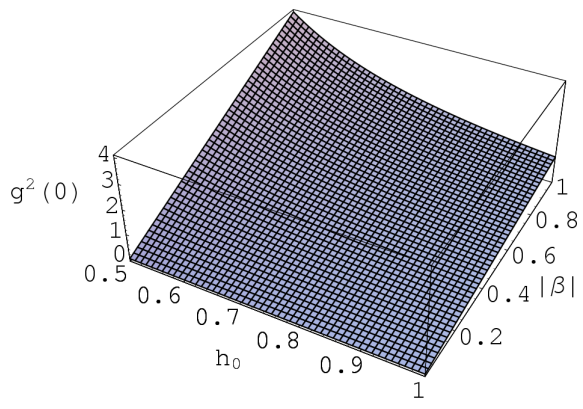

Fig. 7. The second-order coherence $g^{2}(0)$ as a function of $|\beta|$ and $h_{0}$.

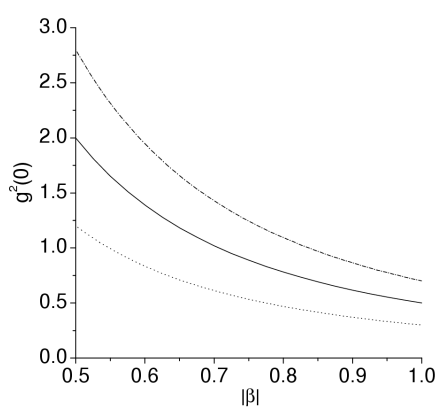

Fig. 8. The second-order coherence $g^{2}(0)$ as a function of $|\beta|$ for three different values of $h_{0}=0.3$ (dotted line), 0.5 (solid line) and 0.7 (dashed-dotted line).

\section{Conclusion}

In summary, we study the nonclassical properties of two kinds of para-Bose superposition states. It is found that these states exhibit squeezing, amplitude-squared squeezing and antibunching effect in some situation. These nonclassical properties are strongly dependent on the para-Bose parameter $h_{0}$.

\section{Acknowledgments}

This project was supported by the National Natural Science Foundation of China (grant no. 10774131) and the Foundation of Educational Commission of Zhejiang Province of China under No. 20060800.

\section{References}

[1] K. Wódkiewicz, P.L. Knight, S.J. Buckle, S.M. Burnett, Phys. Rev. A 35, 2567 (1987).

[2] B.C. Sanders, Phys. Rev. A 39, 4284 (1989).

[3] J. Janszky, A.V. Vinogradov, Phys. Rev. Lett. 64, 2771 (1990).

[4] D.C. Tsui, H.L. Stromer, A.C. Gossard, Phys. Rev. Lett. 48, 1559 (1982).

[5] H.L. Stormer, A. Chang, D.C. Tsui, J.C.M. Hwang, A.C. Gossard, W. Wiegmann, Phys. Rev. Lett. 50, 1953 (1983)

[6] B.I. Halperin, Phys. Rev. Lett. 52, 1583 (1984).

[7] M.V.N. Murthy, R. Shanker, Phys. Rev. Lett. 73, 333 (1994).

[8] F.D.M. Haldane, Phys. Rev. Lett. 67, 937 (1991).

[9] H.S. Green, Phys. Rev. Lett. 90, 270 (1953).

[10] O.W. Greenberg, Phys. Rev. Lett. 64, 705 (1990).

[11] O.W. Greenberg, A.K. Mishra, Phys. Rev. D 70, 125013 (2004).

[12] M. Hillery, Phys. Rev. A 36, 3796 (1987). 